\title{
Methods of visual graph-analytical presentation and retrieval of scientific and technical texts
}

\author{
N.V. Maksimov¹, O.L. Golitsina², K.V. Monankov3, A.S. Gavrilkina4 \\ National Research Nuclear University MEPhI (Moscow Engineering Physics Institute) \\ ${ }^{1}$ ORCID: oooo-0oo2-8191-1521, nv-maks@yandex.ru \\ 2 ORCID: oooo-0oo2-3848-4755, olgolitsina@yandex.ru \\ 3 ORCID: oooo-0002-9267-3987,kmonankov@yandex.ru \\ 4 ORCID: oooo-0003-2167-1287, asgavrilkina@yandex.ru
}

\begin{abstract}
The technology of constructing and visualizing a semantic image of the full text of the document represented by the ontology as a system of three systems is offered: functional, conceptual and terminological. Objects and connections of the functional system correspond to the names of entities and relations extracted from the text; to objects of the conceptual system - descriptors of the thesauri of subject areas. The problem of the variable representation of entities at the sign level is solved using the rules for the formation of phrases of different lengths. Functional relationships are classified according to the taxonomy of functional relationships and are used to construct aspect projections of ontologies. As the data model of the ontology, a labeled directed graph is used, which includes nodes and arcs of different types, which makes it possible to formalize operations on ontologies. Constructing a display of set elements of ontology into graph elements in such way that elements of different sets of different systems are distinguishable, recognizable and depicted in different ways, allows to implement the principle of correspondence of the graphic image with the semantics of the visualized data.

Based on the search tasks typology, metaphors for visualizing the ontology graph are proposed: the "pathfinding" metaphor, characterized by the construction of a directed chain of facts, and the "neighborhood analysis" metaphor, which is characterized by the study of the environment (context) of a fact.

The technology and software for the construction and variant visualization of the ontology graph have been developed.

Examples of using the proposed models for information retrieval through document texts are given.
\end{abstract}

Keywords: semantic search, text processing, graph representations of ontologies, visualization of ontology graphs, visualization metaphor.

\section{Introduction}

The article discusses the issues of the interactive use of graph forms of ontological representations of texts in the tasks of information support by means of documentary information retrieval systems (IRS) of one of the most complex types of human activity scientific research - the process of developing new scientific knowledge, as a result of which new facts are established and the subsequent their generalization.

Traditionally, the problem of information retrieval is formulated as the problem of forming a sample of documents from a certain collection in accordance with the information needs, and the main indicators of the effectiveness of the search process are accuracy, completeness, and efficiency. However, in reality, a person, as a consumer of information, 
does not need a sample of relevant documents and not even their full texts, but a solution to the problem of his main activity, the description of which can be fully or partially contained in the documents found.

Often not a single document contains a complete description of the solution in an explicit form, and a person is faced with the task of forming an image of a solution from fragments of solutions to similar problems available to him. In other words, solving the problem of the main activity requires preliminary formation of the image of the solution. This situation is especially characteristic of scientific or engineering problems, design tasks, etc.

Considering the use of automated documentary IRS as an activity that replaces the main human activity, the task of synthesizing new knowledge can be presented as the task of forming a solution image as a result (and by) constructing ${ }^{1}$ a single text from text fragments of the relevant documents. Such a text (in the form of abstracts, explanatory notes, scientific articles, etc.) represents an image of solving the problem of the main activity ${ }^{2}$.

Knowledge, as an object of human activity, is quite adequately represented by ontological means, since such means, according to $[1,2]$, reflect not only the immanent and situational connections of the subject area (SbA), but also the relationship between the concepts and categories of the tool of knowledge and, in including the language.

In this sense, ontologies can be a "polygon with contour maps", where the user implements the trajectory of both informational and subject search: understanding the expediency of using key concepts is provided by the visualized contexts of these concepts and the construction of paths between them - possible meanings.

At the same time, interactive visualization of the semantic graph of the document ontology allows using the graph as a tool for navigating through the document material, since makes it possible to operate with context-specific subgraphs and transition from graph vertices to text fragments. In the limit, an ontology graph formed from texts in natural language, can serve as a tool for constructing a set of images (alternatives and additions), which together make it possible to solve the user's pragmatic problem. And the study (analysis and synthesis) of an ontology graph as a semiotic object (more precisely, a system) containing many interrelated facts reflecting the meaning of the original text within the framework of the language and conceptual SbA schemes, allows checking the set of facts for consistency, as well as finding implicit (not directly reflected in the source text) facts and connections.

This approach is consistent with the following explication Ch. S. Peirce of the cognitive process [3]: "Effective reasoning is a living process, learning which breaks down disciplinary barriers. Logic deals not with the forms of thought or word, but with general scientific principles that turn reasoning into a self-controlled process, effective for achieving the goal of scientific research. Reasoning cannot be reduced to purely symbolic transformations, but involves observing diagrammatic iconic representations. This observation leads us to setting up an experiment on a graph. Namely, that first we duplicate some parts of the graph, then we erase some of its parts, i.e. hide some part of the statement from observation. We observe the result of this experiment - this is our deductive conclusion.

And this approach - interactive visualization and transformation of semantic graphs as ontological images of documentary information - makes it possible to practically implement the long-formulated requirement: “... the documentary IRS should be organized in such a way that a person could, as it were, explore the search array, changing the wording of the search prescription depending on intermediate search results" [4].

As can be seen, the ideas were formulated a long time ago (especially regarding the pace of development of the IT sector), but their implementation required not only an increase in capacity, IT capacity and the use of effective visualization tools, but also the identification of

\footnotetext{
${ }^{1}$ Such a construction is carried out in accordance with some goal, methodological scheme and criteria for evaluating the result.

${ }^{2}$ At the same time, the person formulates the decision itself in his mind.
} 
fundamental provisions connecting the concepts of such methodologically not so close areas as informatics, linguistics, activity, psychology.

In the article in the context of the main provisions of the information retrieval will be discussed:

— models, tools and technologies for constructing semantic search images based on full texts of documents, providing the possibility of formal analysis and synthesis of graph structures that correspond to cognitive situations;

- models and tools of variant visualization, providing the possibility of reducing the graph's size to an operable level and presentation in accordance with the chosen cognitive metaphor;

- cognitive-like search tools on full texts, which provide controlled manipulation of graph components and corresponding text fragments due to the operations of semantic scaling and construction of aspect projections.

The models and tools for variant visualization of the ontology graph presented in the article are based on the basic principles of constructing visual models [5-7]:

- the principle of correspondence to the task solved by the user, which, in particular, corresponds to the provisions of the correspondence of the graphic image of the semantics of the visualized data to the individual characteristics of perception;

- the principle of ensuring the dynamism of the display;

- the principle of minimizing the time spent on data analysis, including through the choice of optimal algorithms;

— the principle of integrity (consistency) of presentation;

— the principle of independence of levels - physical, logical and presentation.

\section{Building a Semantic Search Image of the Full Document}

\subsection{Basic Provisions of Information Retrieval}

1. The concept of information retrieval is always, in one way or another, associated with a process that has an uncertainty of the outcome, and, if it is a controlled search process, with a choice, which, in turn, is built on the basis of comparing data obtained from outside with available knowledge. Uncertainty (incompleteness and inaccuracy) of the choice is due to successive transformations (understanding - expression - formalization) in the chains "knowledge - information - document - search image of a document" and "problem situation - task - question - search image of a query" linked by means of the IRS, each of which brings its own uncertainty.

2. Due to the principles of organizing the computing environment, the search at the physical level is reduced to an operation (or their sequence for a composite query) of full or partial comparison of a given term (more precisely, a substring) with terms (indexes) of a database. That is, it is necessary to understand (and take for granted) that the retrieval mechanism of the IRS do not have the means of thinking out, guessing or interpreting the specified term.

Note that such tools, and in particular, query expansion technologies (using thesauri, linguistic processors, statistical links, etc.) belong to the level of logic, since they are defined and depend on the characteristics of the conceptual-sign SbA system. But the main thing is that their use in automatic mode in practice rather worsens the integral indicators of search efficiency. Somewhat higher efficiency can be achieved in the interactive mode of using lexicographic reference for subject-oriented vocabulary selection. But again, this will be a choice from a stable and predetermined set, and probably not containing new or alternative vocabulary.

3. The general basis of information documentary retrieval is coordinate indexing - a way of expressing the main semantic content of a document or query in the form of a set of keywords (terms), and it is initially assumed that the keywords are not related to each other, 
but a separate term and document corresponds point in the $n$-dimensional semantic space, which, in fact, fully corresponds to the binary form of information representation in the computing environment.

4. In the aggregate human-machine information system "source of information - IRS consumer of information" IRS essentially (as a result - physically) plays the role of a switch. The source (document) will be transferred to the consumer if his search image meets the criterion of formal relevance used by this system. But both the search image of the document and the search image of the query are descriptive expressions - images of knowledge (implemented and sought), built using a language that allows the conclusion of a comparison result by calculating the value of a certain measure of proximity and correlating it with a threshold value. The most common and appropriate language is the information retrieval language (IRL) of a descriptor-type, whose vocabulary is represented by a set of terms, and the grammar reflects the method of constructing a search image by coordination (interconnection) of terms [4].

At this case, in general case, terms can be both the entity names and the relationship names, and coordination can be implemented in a positional (word combinations, phrases) or key (using relators - linking operators or relationships) method.

Accordingly, coordination can have a different depth of meaning expression:

- terms combinatorially set the characteristic properties of the described object (obligatory/optional, interchangeability, associativity, etc.), which corresponds well to Boolean algebra, when the meaning is determined by a predicate in the form of a logical formula on a set of terms;

— the terms of the search image are presented "in the context", which allows concretizing the meaning;

- the terms of the search image are presented "in sequence", allowing to present (form an image) the meaning and the course of solving a practical problem.

5. In general, the search process is built according to a simple "request-response" scheme and includes three main technological operations: the formation of a request by the user, the formation of the issue by the system, and the assessment of the relevance of the issue by the user3.

However, the following features need to be taken into account. First, the search process will be iterative and not one-act. Second, when adapting a query expression, the objects of three spaces must be semantically linked: mental, operational/interface, machine. In this case, it will be the formation of a query expression that will be decisive, which essentially boils down to the choice of terms and possibly their connection. Such a choice is made by the user, referring either to his own consciousness (knowledge of the vocabulary SbA), or, if the system is interactive, choosing from technological objects generated by the system. Such technological objects can be linear ordered structures (database dictionaries, vocabularies, etc.) or more complex semantic structures (thesauri, semantic networks, cognitive maps, etc.). It is obvious that navigation through such complex and voluminous structures will be no less difficult than the content of the documents themselves. Therefore, for the effective use of such structures, it is necessary to have a means of ordering them, subject-specific articulation, as well as navigation and display control.

6. Search interaction of a person with a machine has its own features. The user identifies and recognizes objects of the practical problem being solved in a certain context. Identifies consists in identifying found objects with available knowledge "in general", and recognition in identifying individual "useful" properties. In the case when a certain practical problem is being solved, the displayed semantic network should be directed (from the initial positions to the "answer") and represent ideally an algorithm for this solution. In the case of the

\footnotetext{
${ }^{3}$ It should be noted that the ultimate goal of the search is not only to solve the problem using the information found, but also the confirmation of the completeness of the final output (confidence that there are no other, alternative ways and solutions). And this is a separate branch of technology.
} 
information and analytical tasks (preliminary research, search for hypotheses or possible solutions), the composition of the elements, structure (blockiness, interconnection), content (nature of entities and relationships), form (ordering and type of presentation) is usually analyzed.

In addition, physically (spatially) the process of sequential perception/understanding (selection of an element, its identification and binding) is realized using some (familiar or special) scheme. For example, "left-to-right" in the case of continuation, expansion of the area: "top - down" or "deep" in case of clarification or detail.

\subsection{Datalogical Model of the Semantic}

According to [8], a fact in the philosophy of science is a special kind of sentence, fixing empirical knowledge, statement or condition that can be verified, and the meaning of a fact is beyond of the fact itself and determines its place in some integrity.

On the basis of this definition, the following types of information components can be distinguished:

- elementary fact is an image that records a certain state of a separate interaction of a pair of entities, where the essence is a concept, object, subject, etc., serve as an entity and the communication is represented by relationship (attitude);

- situational fact is an elementary fact in which both entities (or one of them) are additionally determined by the circumstances of the entity's participation in the interaction a specific situation; thus, a new named entity is formed, including a set of elementary facts;

- completed fact (statement, assertion, description) is a network of elementary and/or situational facts, forming an integrity, correlated with the information request, and thus forming a meaning.

A fact can be fixed and exist in different forms, including in the form of text, as a set of signs of a certain language, in particular, natural.

Just like a meaningful text, an ontology graph built for this text can be considered as a set of facts, together expressing some meaning. In this case, an elementary fact in the ontology graph corresponds to a triplet "entity - relationship - entity", and a situational fact corresponds to a triplet, in which one or both entities are represented by a set of elementary triplets that make up the semantic neighborhood of an atomic entity. Such a meta-entity reflects a certain situation; it can be named and supplied with characteristic attributes. The relationship within the framework of a situational fact will have the character of a metaattitude, since connects not atomic entities, but entire situations. The completed fact is represented in the ontology graph by a certain integral construction of triplets, on the one hand, reconstructing the intentions of the creator of the source text, and on the other hand, corresponding to the context of the main activity problem being solved by the user.

The ontological approach allows the semantics of a separate solution described in the document to be represented by a system of concepts and relations, i.e. when searching, it will be possible to use completed semantic constructions. In this case, the ontology graph will represent the technological space of "entry points" into the information array, providing the possibility of a direct transition from the graph vertices to the document text fragments.

In [1] ontology, as a semiotically integral formation, is defined from the standpoint of general systems theory as a set of three interconnected systems $O=<S_{f}, S_{c}, S_{t} \equiv>$, where

$S_{f}$ is the functional system (objects and relationships of reality), which is defined as $S_{f}=\left\langle M_{f}, A_{f}, R_{f}, Z_{f}>\right.$, where $M_{f}$ is a set of objects (entities), $\mathrm{A}_{f}$ is a set of characteristic properties, and $R_{f}$ is a set of functional relationships represented by typed situational connections of an $\mathrm{SbA}, Z_{f}$ is the composition law, i.e. the rules and schemes for ordering objects (for example, the SbA meronomy).

$S_{c}$ is the conceptual system defined as $S_{c}=<M_{c}, A_{c}, R_{c}, Z_{c}>$, where $M_{c}$ is a set of SbA concepts, $A_{c}$ is a set of signs of systematization of the concepts (the SbA taxonomy), $R_{c}$ is a set of relationships (primarily paradigmatic), $Z_{c}$ is the composition law (representation scheme); 
$S_{t}$ is the terminological system defined as $S_{t}=<M_{t}, A_{t}, R_{t}, Z_{t}>$, where $M_{t}$ is a set of terms, $A_{t}$ is a set of properties, $R_{t}$ is a set of equivalence and inclusion relationships, as well as linguistic relationships, $Z_{t}$ is the composition law (grammar);

$\equiv$ is the operation for comparing the elements of different systems at the level of signs, ensuring their identity in the functional, conceptual and terminological systems.

The representation of an ontology at the structural level in the form of graphs allows formalizing operations on ontologies based on graph-theoretic axioms. The main operations in this case are: binary - unions, intersections, projections and unary - scaling of ontologies $[1,9]$.

As a data model of the ontology functional system, a labeled (for whose vertices and arcs the properties $A_{f}$ are defined) oriented 4 graph $G(V, E)=\langle V, E\rangle$ is used, where $V$ is the set of vertices, and $E$ is the set of arcs. The set of vertices and the set of arcs together correspond to a set of elementary facts. Because the same pair of atomic entities can participate in several elementary facts, in the graph $G(V, E)$ for two vertices there can be more than one arc, that is, $G(V, E)$ has the multigraph property.

According to [10], on the sets $V$ and $E$ are defined (can be dynamically constructed):

1. A metagraph formally defined as $M G=\langle V, M V, E, M E\rangle$, where $V$ is a set of vertices, $M V$ is a set of meta-vertices, $E$ is a set of arcs, $M E$ is a set of meta-arcs. Each meta-vertex corresponds to the meta-entity of the situational fact and is a graph $m v_{i}=\left\langle V_{i}\right.$, $\left.E_{i}\right\rangle$, where $V_{i} \subset V, E_{i} \subset E$, and the meta-arc corresponds to the meta-relationship. The metagraph also has the multigraph property, since situational facts may, for example, differ only in metarelationships.

For graph forms reflecting the semantics of texts (and cognition), the presence of metavertices is quite constructive and natural. The meta-vertex corresponds (by its name) to an entity (concept, node, composition, etc.) and acts as an atomic semantic equivalent of the meaning determined by a non-atomic construction (expression).

2. A hypergraph, when a set of hyperedges $W$ is formed on the set of vertices $V \cup M V$, while the rules for specifying a hyperedge are based on the sets $A_{f}$ and $Z_{f}$.

$$
W=\left\{w_{1}, w_{2}, \ldots, w_{n}\right\}, w_{i}=V_{i} \cup M V_{i} \text {, where } V_{i} \subset V, M V_{i} \subset M V ; V_{i} \neq \emptyset \vee M V_{i} \neq \emptyset
$$

The presence in the functional system of an ontology, in addition to the sets of entities and functional relationships, a set of characteristic properties and the law of composition, makes it possible to group entities not only in dynamics, for example, according to the principle of correspondence of the synthesized chain of facts, but also in statics - for example, according to the principle of possessing a common property, according to lexicographic inclusion, etc.

\subsection{The Application of the Projection Operation to Construct the Aspect Representation}

Aspect representation, as one of the forms of a completed fact, representing a certain semantic slice of an SbA, within the framework of a functional ontology system is implemented in the form of a subgraph. The construction of the aspect representation is based on the projection operation, which in [1] is reduced to the operation of intersection of the original $O=\left\langle S_{f}, S_{c}, S_{t}, \equiv\right\rangle$ and aspect $O_{i}=\left\langle S_{f}^{i}, S_{c}, S_{t}, \equiv\right\rangle$ ontologies: $O_{p r o j}=$ $O \cap O_{i}$. Thus, for each aspect representation, its own aspect ontology must be specified (at the level of the functional system).

\footnotetext{
${ }^{4}$ Note that the orientation of the ontology graph (first of all, this applies to the functional system) is determined not only by the orientation of the arcs, but also by the semantic "directionality" reflecting the evolution of the semantic image of the object/result. This means that the name of an individual entity or relationship in the graph will be presented in several "instances". In this case, it can be argued that both concepts and relationships act as linguistic variables, the specific meaning of which is further determined by the situation - a well-defined context (this is also a controlled context: through the setting of an aspect and/or a parameter of conceptual depth and/or breadth), specified by the types of relationships and the nature of related entities. And, since the graph represents a goaloriented process, the order of the vertices must be determined, including the initial and final (target) vertices.
} 
In general, the taxonomy of aspects is used, which (being an object open to extension and modification) specifies a set of possible aspects associated with the relationship classes specific to this point of view. A set of aspects 5 is determined in accordance with the activity model and is set on the taxonomy of functional relationships, whose relationship classes are associated with linguistic constructions in the text [10], supplemented by a variety of structural and linguistic relationships. The latter make it possible to take into account the connections of language (synonymy, paradigmatics), as well as the "constructional" connections characteristic of the setting of properties (name, dimension, parameter value).

Thus, specifying an aspect within a taxonomy is reduced to the formation of a functional system $S_{f}^{i}=\left\langle\emptyset, A_{f}^{i}, R_{f}^{i}, Z_{f}^{i}\right\rangle$ with a non-empty set $R_{f}^{i}$ and, possibly, non-empty sets $A_{f}^{i}$ and/or $Z_{f}^{i}$.

In more detail, an aspect can be specified by its characteristic set of basic concepts (entity names), i.e. $M_{f}^{i} \neq \varnothing$. In this case, only the names of the specified entities will participate in the projection formation.

\subsection{Fact Indexing}

Indexing technologies are the basis for information retrieval. Traditionally (and quite justifiably), the names of concepts (or objects, properties, etc.) extracted from the text are used as search indexes. Such indexes are focused on the use of descriptor-type IRLs, which consider linear search images of documents as operational objects. The semantic power of such languages is limited by the presence of synonymy, polysemy and homonymy in natural language and the lack of means of expressing situational and immanent relationships between real objects, processes, etc., presented at the verbal level in the text.

A semantic classification of the IRL was proposed, as early as [11], on the basis of two classification levels: paradigmatic and syntagmatic. At the paradigmatic level, the classification presents classes of languages without means to express the immanent relationships; languages in which there are means of expressing part of the immanent relationships, and languages with all the immanent relationships of the considered SbA. At the syntagmatic level, language classes lacking means of expressing situational relationships; languages that have the means to express situational relationships, but no means to distinguish them, and languages in which situational relationships are expressed and different.

The formation of an ontology as a search image of a network organization requires:

1. Define a conceptual system of ontology with a set of paradigmatic relationships.

2. Present the text of the document as a set of elementary facts. At this stage, in addition to entity names, situational relationships are formed, which can be typed according to the taxonomy proposed in [10]. The analysis of entity names allows to form additional structurallinguistic relationships based on the recognition of abbreviations, units of measure, division of long sequences according to the rules of natural language, etc., and also define the entity (concepts) names that are entry points into the conceptual system of ontology.

Expression of entity names and relationships names at the sign level allows to index an elementary fact as a triplet - a sequence of signs in which not only names, but also entity types and relationships are presented. Thus, both traditional indexes (by keywords) and indexes representing semantic relationships can be built. The presence of such indexes allows, within the traditional Set Theory model of information retrieval (and by means of the traditional descriptor IRL), to implement the selection of documents taking into account the immanent and situational relationships between entities. In this case, the IRL falls into the

\footnotetext{
${ }^{5}$ Aspect representations are one of the methodological foundations of the synthesis of knowledge. The synthesis of knowledge as a self-organizing process is based on the structural feature of the system - a complex system can be described using a set of relatively independent aspect representations. Moreover, the decomposition process not only separates and binds components, but also the decomposition scheme is formed - a system of characteristic signs of division.
} 
semantic classification in the class of languages that have means of expression (and distinction) of both immanent and situational relationships.

\subsection{Stages for Constructing a Semantic Image of a Document}

The construction of the semantic image of the document as a set of elementary facts that form nodes and arcs of the ontology graph is based on a classical semantic text analysis scheme, which includes the stages of graphematic, morphological, semantic-syntactic and conceptual analysis [12].

At the stage of graphematic analysis, the structural elements of the text (sections, chapters, paragraphs, headings) are traditionally distinguished, the text is split into tokens, which are identified and (if necessary) combined using dictionaries and linguistic rules. Named groups, dates, floating point numbers, abbreviations, units of measurement are identified. The boundaries of sentences are determined by punctuation marks, taking into account the identified specific character sequences.

The task of the morphological analysis stage is to determine the main morphological characteristics (part of speech, gender, number, case) of tokens identified as words.

The stage of semantic-syntactic analysis begins with the removal of morphological ambiguity generated at the stage of morphological analysis. The choice of a single word paradigm is based on the analysis of the contextual environment and the application of the rules of the Russian language.

At the stage of semantic-syntactic analysis, the formation of elementary facts is carried out on the basis of lexical-syntactic templates. The algorithm for forming of elementary facts is based on the presentation of a separate sentence in the form of a linear sequence of segments, each of which is identified as "the name of the subject / object" or "connection (part of the connection)".

For example, the sentence "The lower spacer brackets rest are supported by roller bearings installed on the slab" would be divided as follows:

(The lower spacer brackets) < subject/object name $>$ |

(rest) $<$ connection (part of the connection) $>1$

(on) $<$ connection (part of the connection) $>1$

(roller bearings) $<$ subject/object name $>1$

(installed) $<$ connection (part of the connection) $>\mid$

(on) $<$ connection (part of the connection) $>$ |

(the slab) < subject/object name >

The template description language allows for the triplet $<$ subject $(\mathrm{S})><$ relationship $(\mathrm{L})>$ $<$ object $(\mathrm{O})>$ to specify sequences of sentence fragments that must define (or are part of) each of the parts of the triplet. Many templates can be formed depending on the type of processed texts. For the given example, triplets will be built:

$<$ Bracket lower spacer $(\mathrm{S})><$ support $(\mathrm{L} 1)><\mathrm{On}(\mathrm{L} 2)><$ roller support $(\mathrm{O})>$

$<$ roller bearing $(\mathrm{S})><$ install $(\mathrm{L} 1)><$ on $(\mathrm{L} 2)><$ overlap $(\mathrm{O})>$

Thus, the linear text is transformed into a set of triplets that form the nodes and arcs of the graph of the functional system of the ontology.

At the stage of conceptual analysis, the following tasks are solved:

1. Classification of formed links (relationships) in accordance with the taxonomy of relationships [13] and identification of modal properties of relationships using morphological characteristics and signal words. The modalities can have the following meanings: Accurate (Relevant)/Prospective (possible)/Impossible and Fulfilled/Completed/Expected.

2. Identification of the names of subjects/objects (or parts of names) that are part of the conceptual system of ontology. For such concepts, independent nodes and arcs are formed. The nodes further serve as inputs to the graph of the conceptual system, and the arcs represent the corresponding structural and linguistic relationships. 
3. Identification of parts of the names of subjects/objects that are abbreviations, noun phrases, and the formation of additional nodes and arcs corresponding to structurallinguistic relationships.

4. Identification of the names of subjects/objects (parts of names), which are units of measurement. Additional nodes are created for units of measure and for property names, which are defined according to the taxonomy of properties and units [14]. The property name and corresponding unit of measure are connected by an arc.

5. Formation of arcs of structural and linguistic relationships according to the principle of lexicographic inclusion between the names of subjects/objects.

6. Identification of the subjects/objects names containing the name of more than one entity. Such names are divided into two or more in accordance with the rules for the formation of phrases, additional nodes are created (for example, for the name "The lower spacer bracket", two additional nodes will be formed - "bracket" and "lower spacer"), which are connected to the original nodes by arcs corresponding to structural-linguistic relationships.

7. Calculation of the frequency characteristics of the names of subjects/objects. Calculation of the weight of nodes based on the frequency of occurrence, role and belonging to significant text fragments.

As a result of the sequential execution of the stages, a complete graph of the functional ontology system is formed, which serves as the basis for the semantic image of the document.

\section{Ontology Graph Visualization}

The problem of visualizing the ontology graph is due to the fact that: on the one hand, an elementary fact must have a visual image that makes it possible to distinguish separate entities and relationships, and on the other hand, the visual image of a situational or completed fact must be available for perception in its entirety and fit into the screen space. The ontology of a document full text is usually characterized by large cardinalities of elements sets (it can be thousands of entities and relationships even for a small text), which predetermines the need to create and use tools for selecting and visualizing fragments of the ontology graph. Moreover, such tools should be adequate to the type of problem being solved.

A visualization process any data is aimed at presenting them in a perceived and convenient for visual observation and analysis form. The effectiveness of visualization methods is largely ensured by the meaningful use of the principles of information perception, an adequate choice of the metaphor and the visualization model and compliance with the visualization criteria.

In this article, the visualization metaphor is understood as the mapping of a set of objects in the data space of the original problem to a set of objects in the representation space by transferring the attributes of objects of the first set to objects of the second in an intuitive way $[7,15]$.

Some examples of visualization metaphors are discussed in detail in [7, 15, 16 and 17], in particular, such as the metaphor of the desktop, rooms, buildings, molecules. For objects represented by a graph, the color and sizes of graph elements are often used to create visual markers that allow the user's attention to be focused. For example, for cognitive maps [16] an arc color corresponds to the relationship type, the thickness corresponds to its intensity and the vertex color and size depends on the vertex type or it's belonging to a semantic group.

In the context of the tasks of documentary information retrieval from the point of view of mapping the ontology graph to the situation diagram of the user's cognitive state, two metaphors have been identified: a path search metaphor and a neighborhood analysis metaphor.

The criteria for graph visualization are $[18,19]$ :

— distinguishability of individual elements of the graph (vertices, arcs, marks);

- convenient laying of vertices on a plane in terms of visual perception; 
- acceptable waiting time and, as a consequence, an acceptable (close to linear) computational complexity of vertex layout algorithms;

- the preservation of the mental map of the graph, which sets the requirement for the similarity of styling in the event of a slight change in the graph.

The criteria are influenced by visualization rules, often referred to as aesthetic criteria [16, 18 and 20]:

— minimizing the size of the area occupied;

- uniformity of the vertex distribution on the plane;

- minimization of arc crossings (approximation to planar map);

— minimization of the total length of all arcs;

— minimizing the number of arcs bends

— directionality of arcs ("top-down" and "left-right");

- compliance with symmetry.

However, it is usually impossible to satisfy all the rules. First, because of conflicting rules (for example, minimizing the number of arcs bends can violate the uniformity of the vertices distribution on the plane and lead to an increase in the size of the occupied area) [20]. Second, because of the increasing computational complexity of the algorithms.

In this article, elements of the ontology (elements of the sets of each of the three systems) are associated with the elements of the graph - marked vertices and arcs of different types that characterize the origin of the element. The vertex of the graph is represented by a rectangle and marked with the name of the entity - concept, object name, unit of measure, etc. The vertex size (label font size) is calculated based on the weight of the corresponding name in the text. The vertex color is set depending on the vertex type or the role of the name. The graph arc is represented by a curved line ${ }^{6}$, which allows the arc label and the incident vertices labels to be placed on different horizontals, which in the case of long label names allows reducing the number of their intersections and avoiding a high concentration of intersections. Drawing a curved line requires more computational resources (and therefore more time-consuming) than drawing a straight line, but the resulting graph image makes it easier to distinguish between its separate elements. The graph arcs are labeled in different ways, including the relationship name or the relationship class name, depending on the arc type and visualization parameters.

\subsection{Ontology Graph Visualization Technology}

The visualization technology of an ontology graph built for a natural language text includes the following stages:

- graph elements selection in accordance with the user's task;

- formation of presentation in accordance with the visualization metaphor;

— image formation in accordance with the visualization model.

At the stage of selecting graph elements, filters are used that are set automatically (in the case, for example, of forming an aspect projection) or manually. All filters are divided into three groups: filters of the vertices set, filters of the arcs set, and filters related to the graph as a whole. Filters of the vertices set assume the selection of vertices by entity name, vertex type ${ }^{7}$, location in the source text, term role ${ }^{8}$, weight 9 . After applying the vertex filters, the graph will contain the vertices that satisfy the filter conditions and the arcs incident to such vertices. Filters of the arcs set assume the selection of arcs by relationship name, $\operatorname{class}^{10}$,

\footnotetext{
${ }^{6}$ In this article, the open source visualization library "vis network.js" [21] is used to draw the graph. The library implements various methods of drawing arcs with curves. The authors chose the method of depicting arcs with quadratic Bezier curves for aesthetic reasons.

${ }^{7}$ Vertex type characterizes the origin of the corresponding name: from text, from thesaurus, from taxonomy of properties and units, part of a longer term and uniting vertices.

${ }^{8}$ The role of the entity name is defined according to a functional model similar to IDEF0-model.

${ }^{9}$ The weight of the vertex is calculated based on the frequency of occurrence, role, belonging to significant text fragments.

${ }^{10}$ The relationship class is determined in accordance with the taxonomy of functional relationship classes [13].
} 
modality, location in the source text. After applying the arcs filters, the graph will contain arcs that satisfy the filter conditions and the vertices incident to them. Filters related to the graph as a whole allow specifying additional conditions for selecting vertices and arcs. For example, leave in the graph the vertices that do not satisfy the vertex filter, but are included with those vertices in one connected component.

The visualization metaphor provides the mapping of the ontology graph to cognitive state situation diagram and the user's goal (finding a solution to a practical problem or analyzing a problem situation).

The visualization model (data presentation) is specified by a set of rules for the formation of visual objects and their graphic attributes, such as color, shape, size, and must provide a variant geometry of the generated set of arcs and vertices. In accordance with the visualization model, the graph of the vertices are laid on a plane i.e. calculation of their coordinates in space.

Thus, the visualization technology provides the construction of a dynamic graphical form that provides the user with the following interactive interaction possibilities:

— viewing the properties of vertices and arcs;

— moving vertices on a plane;

— changing, deleting and creating new vertices and arcs;

- approximation and scrolling of the graph, allowing to interact with separate fragments of the graph with varying degrees of detail;

- search for vertices and move focus to the found vertices;

- transition from the vertices to the corresponding text fragments;

— building a path between two vertices, if such a path exists;

— building a neighborhood of the vertex;

- building of aspect projections of the graph;

- selection of graph elements in accordance with filters;

- union and intersection of graphs.

The stages of the technology correspond to the traditional approach to data visualization, which involves performing the following stages: setting the source data, filtering, mapping and rendering - which are collectively called the visualization pipeline [22]. The correspondence is shown in Fig. 1.

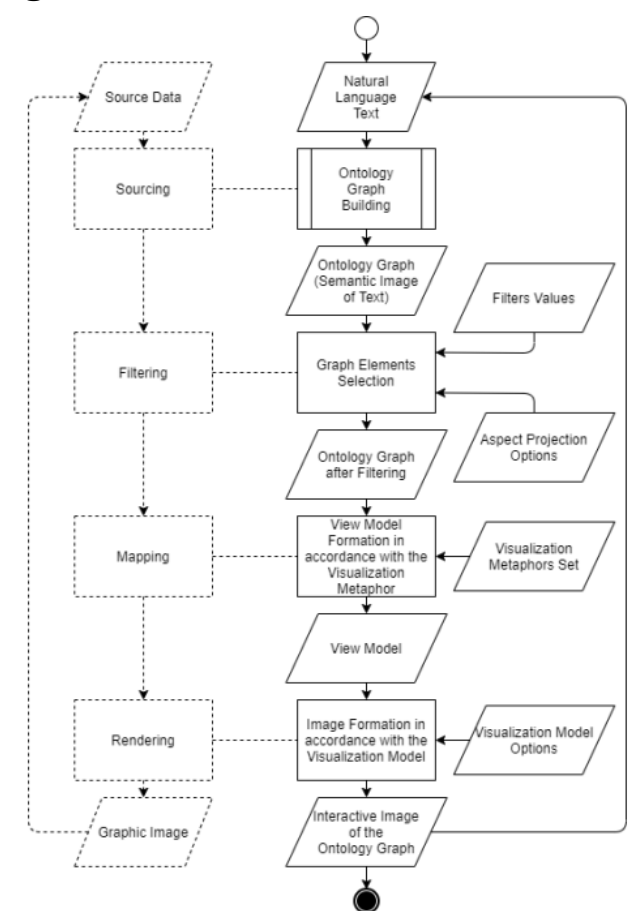

Figure 1 - Ontology graph visualization technology in accordance with the stages of the visualization pipeline [22] 


\subsection{Visualization Model}

In this article, the visualization model of the ontology graph corresponds to the method of drawing a separate element of the ontology and algorithm for layout a set of elements on a plane.

The typology of algorithms for layout the vertices of a graph on a plane is given in [23] and includes the following:

- algorithms using graph theory and graph algorithms;

— algorithms using force models (for example, spring), thermodynamics models (simulated annealing), bioinformatics model (genetic algorithm) and other simulations;

— heuristic algorithms;

- combinations of the above algorithms.

In general, the use of different display options when solving a user problem allows looking at its possible solutions from different angles, in different aspects, which provides complementarity of approaches and allows increasing the perception and depth of understanding of the problem.

For an ontology graph, it is proposed to use the following algorithms for layout vertices on a plane:

- layout vertices using the Barnes-Hut force method;

- layout vertices in the order of use (appearance) of entity names in the text;

- layout vertices in accordance with the significance of the paths (long path or the total weight of the vertices); IDEFo).

- layout vertices in accordance with some scheme (for example, functional model

Force algorithms are based on physical analogies, however, in practice, force algorithms are unacceptable in terms of the construction time for layout large graphs [24], and therefore, their different modifications are often used.

For example, the Barnes-Hut method known in astrophysics, formulated in terms of solving the gravitational $n$-body problem, allows accelerating graph layout by approximating repulsive forces.

The following models of ontology visualization are considered in this article:

— visualization model of the shortest path between two entities, providing a representation of a chain of elementary facts;

- path visualization model based on layout with ordering by significance, where the path length or the total weight of vertices is used as the significance;

- entity neighborhood visualization model based on the Barnes-Hut force-based vertex layout.

Let us consider in more detail the model of path visualization with order in importance. The following algorithm has been developed for layout vertices:

1. Search for a set of paths between all the vertices of the graph (for example, using the Floyd-Warshall algorithm), the length or total weight of the vertices of which exceeds the specified threshold value (by default, these parameters are equal to 1). The search is carried out taking into account (or not) the orientation of the arcs.

2. Sorting the set of paths in descending order of path length or total weight of vertices.

3. Sequential arrangement on a plane (assignment of coordinates to the vertices) of paths in such a way that the most significant paths are located higher, and the following features are taken into account:

- each path fits from left to right, based on the order of the vertices that make it up;

- vertices that are part of multiple paths are not duplicated, and their incident arcs are attached to the first known instance of a vertex (such arcs reflect connections between paths). 
4. Analysis of the density of subgraphs and the connectivity of graph paths. The connectivity between the two paths is calculated from the number of common vertices and by the number of arcs between the vertices of the first and second path. The paths that are more connected are adjacent. The densest subgraphs and connectivity components are separated from each other on the plane. The hanging vertices of the most significant paths are approaching them in the plane.

The fourth step of the algorithm is aimed at reducing the size of the occupied area, reducing the number of intersections of arcs and reducing their length. However, due to the high computational complexity that results in longer waiting times for visualization, this step is not mandatory.

A model for visualizing an entity's surroundings provides a representation of all elementary facts extracted from the text in which the respective entity's apex is involved. Sequential application of the model allows choosing the next vertex for analysis and thus choose a direction and form a semantic neighborhood. In this way, the researcher forms an idea of the object sought, whose depth and completeness are determined by the content of the text.

So, consider the algorithm for working with the Neighborhood Visualization Model:

1. Selecting of vertices of an ontology graph from a given entity name (may correspond to an expression of a search query), resulting in some subgraph containing the vertices $\left\{v_{1} \ldots\right.$ $\left.v_{n}\right\}$.

2. Construction of a neighborhood of a vertex $v_{i} \in\left\{v_{1} \ldots v_{n}\right\}$, resulting in a subgraph consisting of the vertices $\left\{v_{1} \ldots v_{n}\right\}$ and related to $v_{i}$ in some neighborhood the "radius" of the neighborhood defines the user) to a vertex $v_{i}$ vertices $\left\{v_{n+1} \ldots v_{m}\right\}$.

3. Executing p. 2 of the algorithm for any other vertex in the set $\left\{v_{1} \ldots v_{n}, v_{n+1} \ldots v_{m}\right\}$.

\section{Information Retrieval on Ontology Graphs}

The semantic search task can be reduced to an iterative sequential solution of two tasks: a classic information retrieval task and in-depth analysis of the found documents, using the ontology graph of the document as an interactive text and conceptual navigation tool. The generic search scheme is shown in Fig. 2. The traditional scheme is supplemented by the construction stages and analysis of the ontology graph of the full text of document,combining text fragments (information blocks) and evaluating the results of the combination.

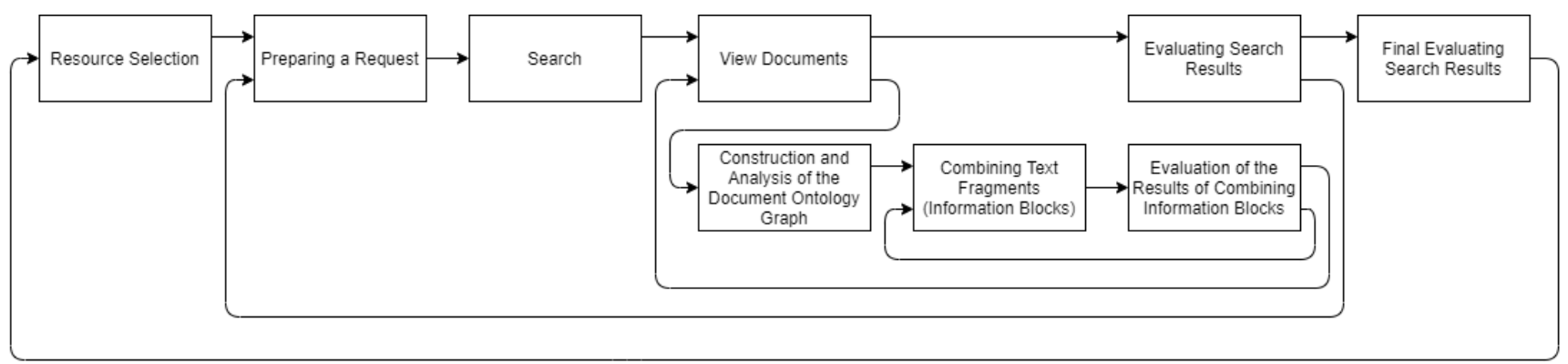

Figure 2 - Generalized search scheme

Visual analysis of the document's ontology graph allows detecting paths or connectivity components relevant to the user's search needs. The transition from graph vertices to source text fragments and the combination of such fragments allows new knowledge to be discovered, as well as checking for consistency of existing ones.

\subsection{Types of Information Retrieval Tasks on Ontology Graphs}

The presentation of the search results should be adequate to the nature of the task to be performed by the user's task, so that providing the person with a better perception, 
depending on the type of task to be solved, is an important aspect of information visualization.

The information retrieval tasks can be divided into two types - the tasks of finding a solution to the problem of the main activity and the tasks of an information-analytical nature, such as preliminary research, the search for hypotheses or possible solutions.

The first type tasks involve finding a solution that can always be represented by a process, that is, a directed sequence of events and actions on objects. This makes it necessary to present the search results in the form reflecting the focus (from the starting point to the "response") of the decision and, in the ideal case, represent ideally the algorithm of this decision. The "pathfinding" metaphor is suitable for this kind of tasks, which involves sequencing points, corresponding to objects, events, actions, expressed concepts (chains of elementary facts), from basic concepts to concepts in the context of a potential solution to the main activity problem.

The form of presentation of the visualization results in the ontology graph corresponding to this metaphor is the path from the vertices of the initial data (reference concepts, query terms) to the vertices containing the solution concepts.

For information-analytical tasks, the metaphor of the search for a neighborhood can be used, which involves visualizing the context of the reference concepts. Grouping around reference concepts allows the user to view the neighborhood, deepening into the exploration topic.

Thus, information retrieval on ontology graphs is reduced to the following schemes:

- search for a chain of facts corresponding to the fragments of the source text, in the aggregate containing the solution to the problem of the main activity;

- search for the neighborhood of an elementary (situational) fact, where the reference concept (the vertex of the graph) is used as a starting point;

- a combination of the first two schemes.

The "finding a chain of facts" scheme involves the selection of vertices, lined up in a directed or undirected chain (analogy - finding a path between vertices) from the original elementary fact to the target one (analogy - finding causal relationships).

The "neighborhood search" scheme implies the selection of vertices in the neighborhood of the original elementary fact (analogy - breadth search in the graph), and the construction from selected vertices of the complete construction - a completed fact (analogy - a snowflake scheme).

The combination of "finding a chain of facts" and "neighborhood search" schemes involves their sequential or combined application.

To solve these problems, the program "Service for visual ontological analysis of scientific and technical texts" [25] has been developed, implementing the models and visualization technology considered in the article. The program includes a language parsing component of the text using the methods of the «xIRBIS» [26] documentation and analysis system and the lexicographic database [27].

For the drawing of the ontology graph, the open source visualization library "vis network.js" [21] is used. The interface fragment of the developed program is shown in Fig. $3^{11}$. The program can function independently (in the web server mode) and can be used for semantic text analysis, as well as a part of the "xIRBIS" system [26] which allows to apply the developed models and technology directly in the process of information retrieval.

\footnotetext{
${ }^{11}$ The program is designed to work with texts in Russian. The interface is also designed in Russian.
} 


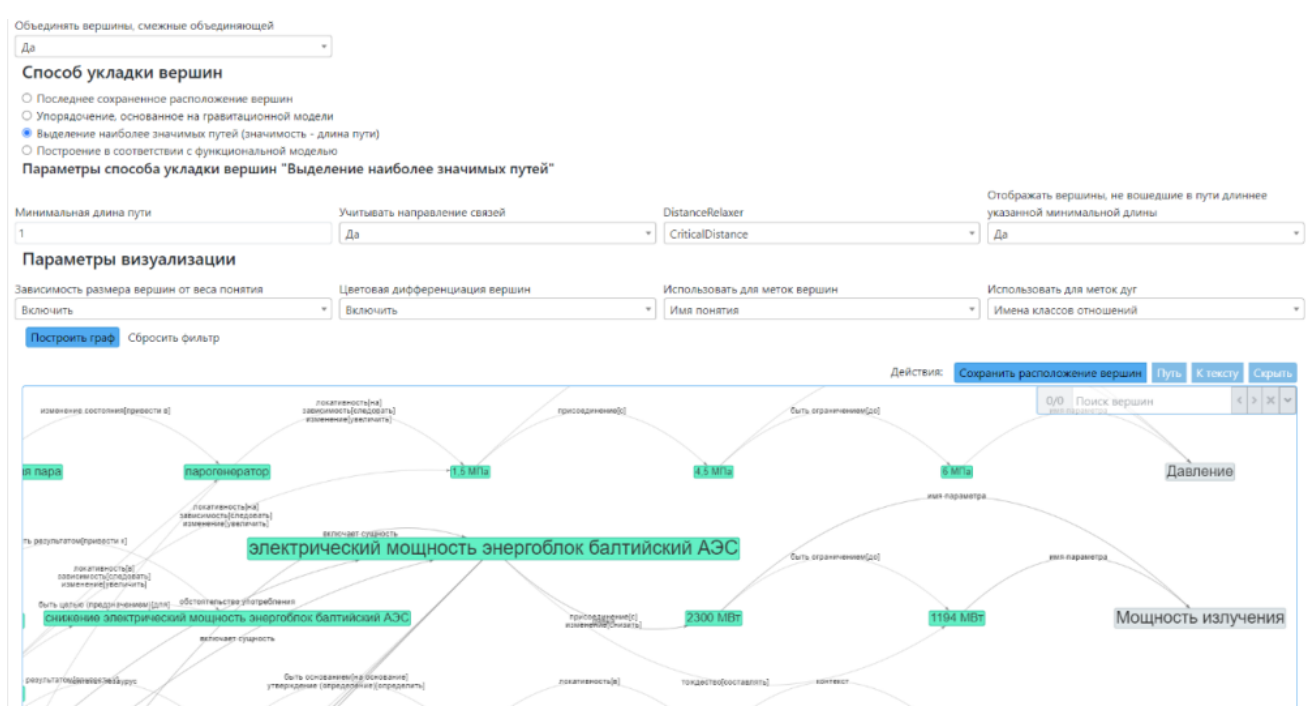

Figure 3 - Interface fragment of the Scientific and Technical Visual Ontological Text Analysis Service $^{12}$

\subsection{Finding a Path on an Ontology Graph}

Let us illustrate the application of the developed tools with the example of the problem of incomplete capacity demand of the Baltic nuclear power plant under construction. As a result of information retrieval in the information resource on the problem, documents [28, 29.30] were found, the relevant fragments of which are combined into a new text. The ontology graph is built on this text ${ }^{13}$, which, due to its large volume, is not fully given here. A fragment of the ontology graph containing the vertex "Power" is shown in Fig. 4.

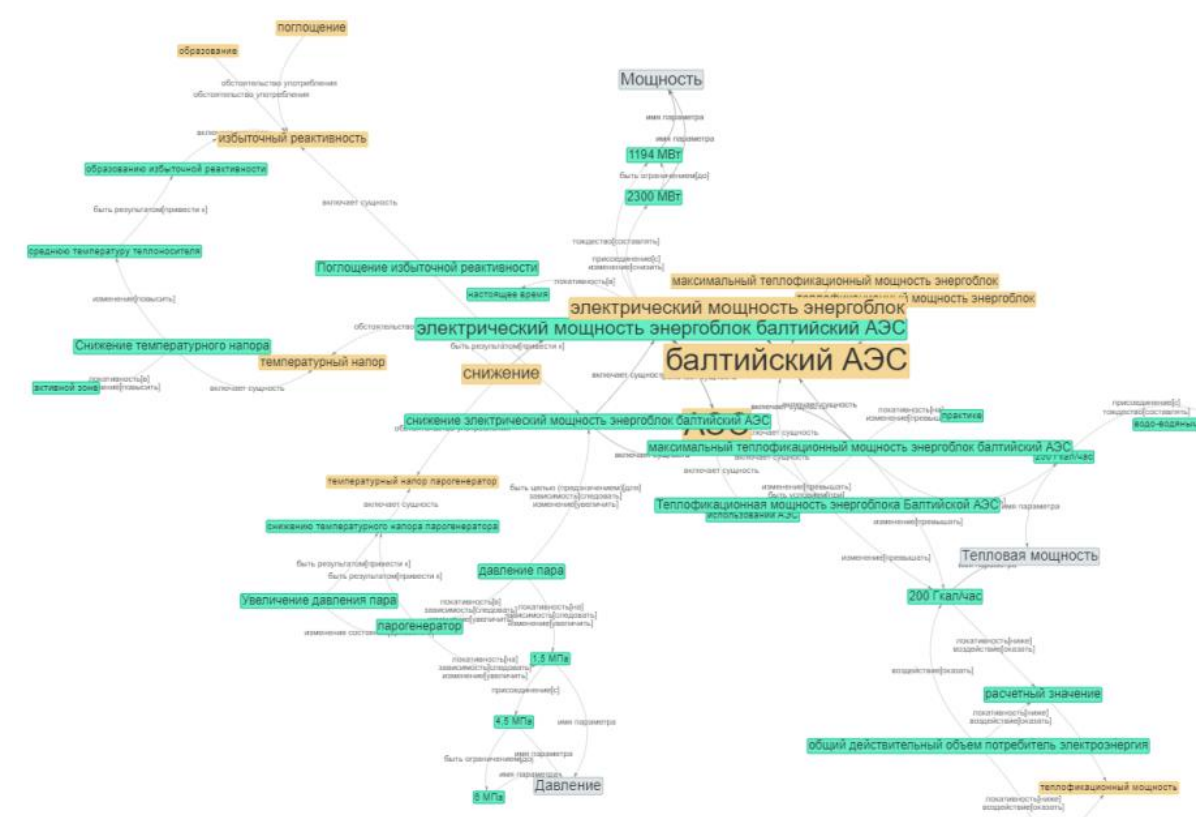

Figure 4 - Fragment of the ontology graph of the text "The problem of incomplete capacity demand of the Baltic NPP under construction" with layout vertices using the Barnes-Hut method

\footnotetext{
12 The following image agreement is then used. Green vertices have type «from the text», blue - "from the thesaurus", grey - "names of properties", yellow - "parts of longer terms". The names of the classes of relations are used as labels of the arcs of functional relations, next to each class name in square brackets is the normalized linguistic construction by which the class was defined. The label «parameter name» marks an arc that links a value from the text to the corresponding property name from the taxonomy of properties and measurement units.

${ }^{13}$ A graph can also be constructed by combining relevant graph fragments of ontology of document text.
} 
As a result of finding vertices in the graph by the query "Power", 12 vertices were found, one of which (the gray vertex in the upper part of Fig. 4) is extracted in accordance with the taxonomy of properties and units of measure [14] due to the presence of the vertices " 1194 MW" and "2300 MW", containing the unit of measurement megawatts. Other properties extracted similarly are the vertex "Pressure" (gray vertex at the bottom of Fig. 4) and "Heat rate" (gray vertex at the right side of Fig. 4). Let's build the shortest path between the vertices "Pressure" and "Thermal power" (see Fig. 5, the path is highlighted in red) in order to trace the relationship between the corresponding parameters.

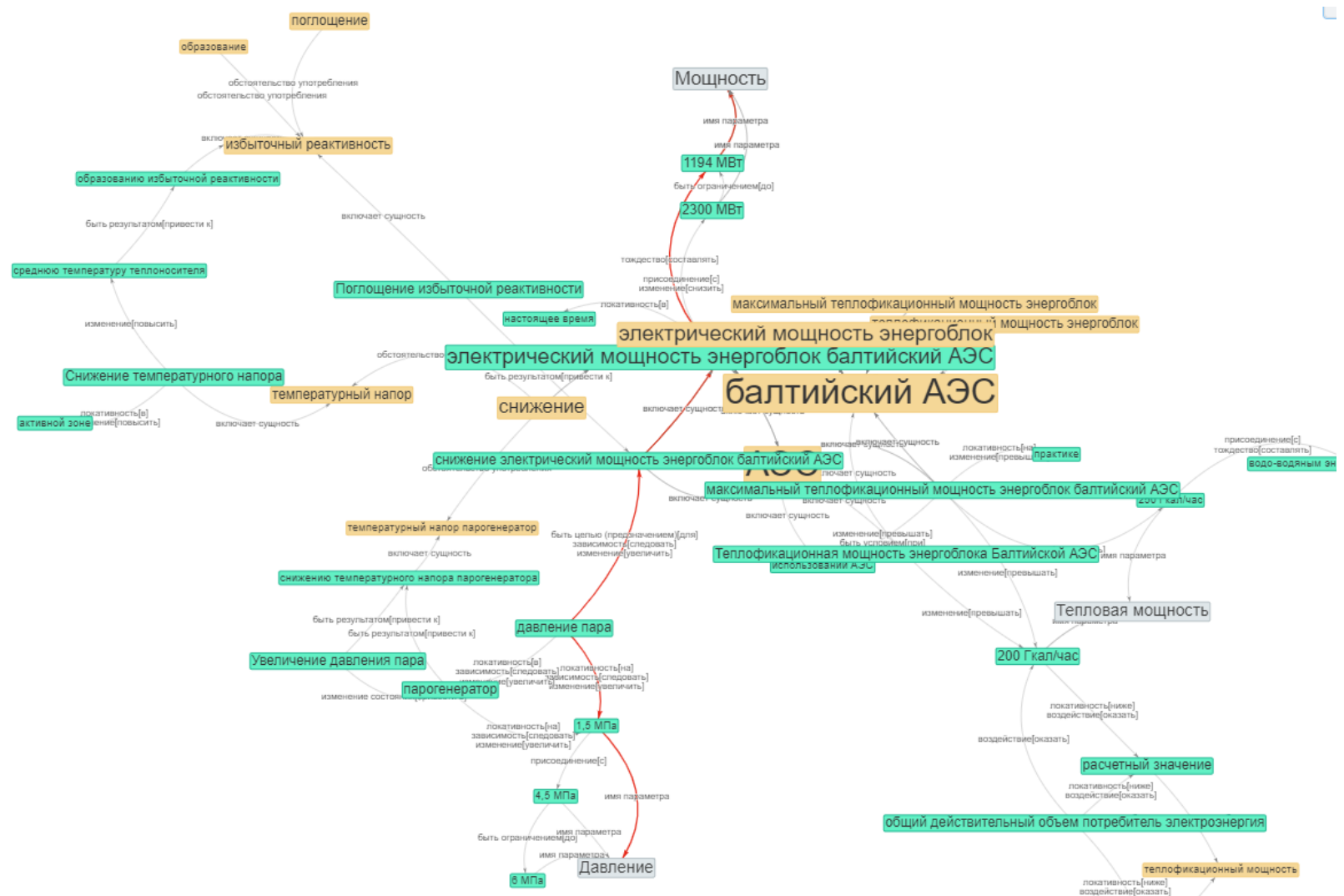

Figure 5 - Fragment of the ontology graph of the text "The problem of incomplete demand for the power of the Baltic NPP" with the graph layout using the Barnes-Hut method and the shortest path (highlighted in red) between the vertices "Pressure" and "Power"

The path (see Fig. 5) contains the elementary fact "vapor pressure" - "to be the goal (destination) [for] dependence [follow] change [increase]" - "reduce the electrical power of the power unit of the Baltic NPP". Let us consider further the elementary fact "vapor pressure" - "locality [in]" - "steam generator". It follows from these two elementary facts that the parameters of the steam generator vapor pressure and the electric power of the power unit are related. Now it is necessary to establish the nature of the connection.

Next to the red path notice the "decrease" vertex (yellow vertex in the center of Fig. 5, to the left of the red path). This vertex is visually differs in color and font size, which is a visual marker and indicates the possible significance of the vertex.

Let's build the shortest path between the "Pressure" and "Power" vertices through the intermediate "decrease" vertex (see Fig. 6, the path is highlighted in red). 


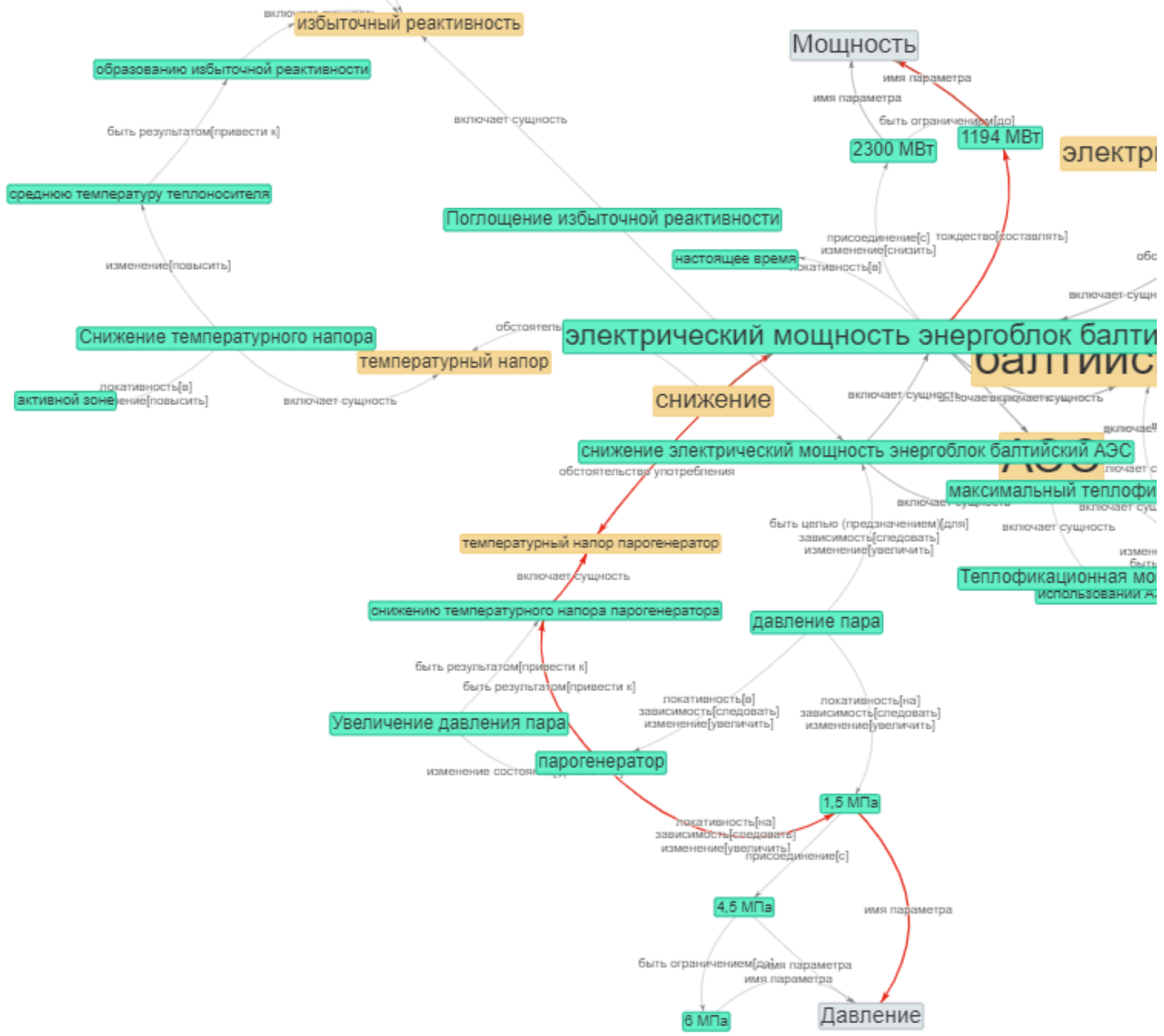

Figure 6 - Fragment of the ontology graph of the text "The problem of incomplete capacity demand of the Baltic NPP under construction" with the laying of peaks using the Barnes-Hut method and the shortest path (highlighted in red) between the vertices "Pressure" and "Power" through the intermediate vertex "decrease"

Let us consider the vertices on the constructed path (Fig. 6) in the context of adjacent vertices and see the following chain of elementary facts: "increase in steam pressure" "change of state [bring to]" - "steam generator" - "be the result [lead to]" - "decrease of steam generator temperature pressure". That is, an increase in the pressure of the steam generator leads to a decrease in the temperature pressure. Further on the way "temperature pressure of the steam generator" - "circumstance of consumption" - "decrease" "circumstance of consumption" - "electric power of the power unit of the Baltic NPP". Let us consider in more detail the circumstances of the use of electric power of the power unit of the Baltic NPP in the context of the reduction. The vertex of the "descent" has another arc "use circumstance", an incident vertex of the "temperature pressure", which through a chain of elementary facts is related to the vertex of the «redundant reactivity».

Let' s build the shortest path between the vertices "Power" and "Pressure" through the intermediate vertices "temperature head" and "excess reactivity" (see Fig. 7, the path is highlighted in red). 


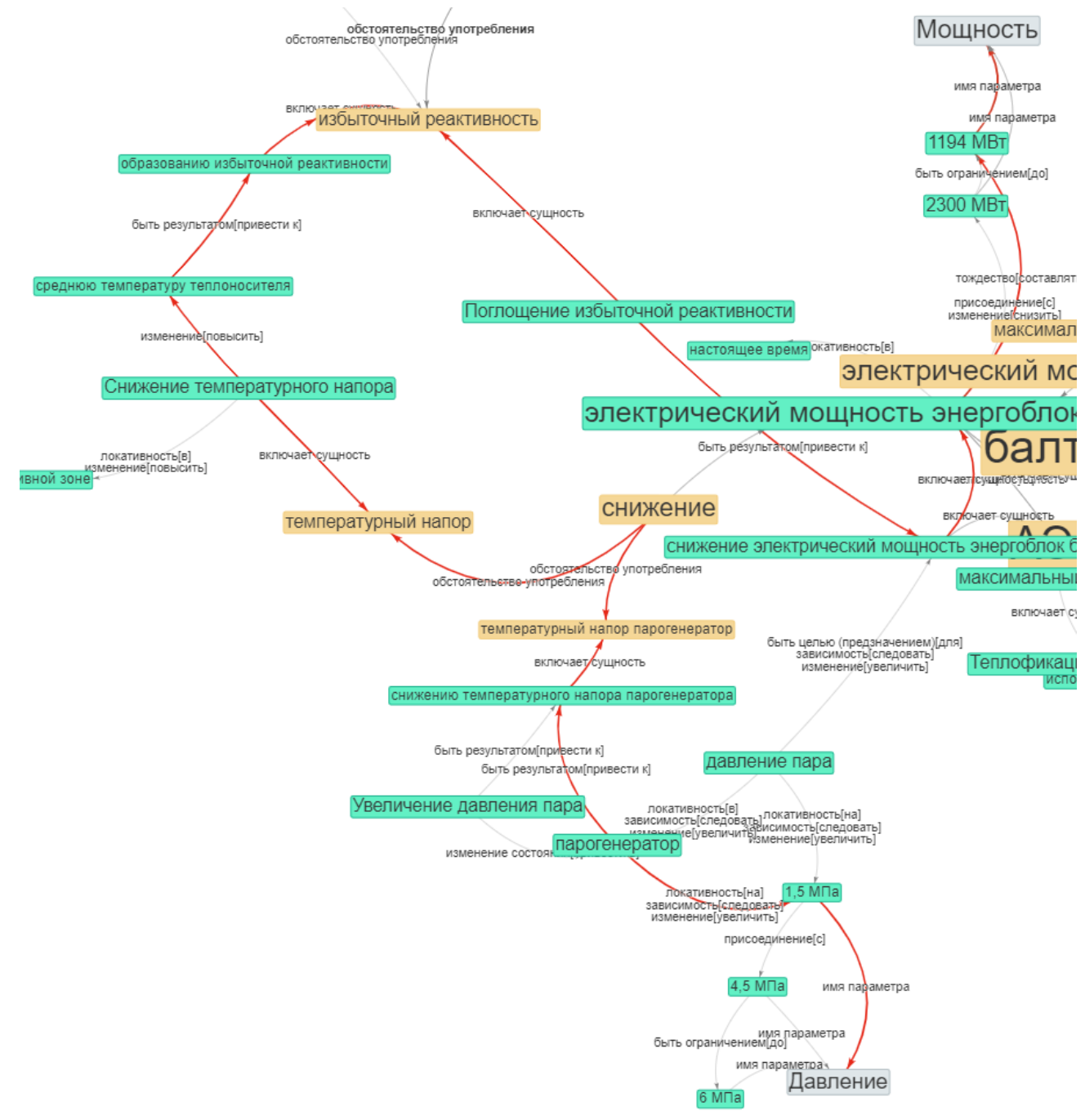

Figure 7 - Fragment of the ontology graph of the text "problem of incomplete demand capacity of the Baltic NPP under construction" with the laying of vertices by the Barnes-Hut

method and the shortest path (highlighted in red) between the vertices "Pressure" and

"Power" through the intermediate vertices "temperature pressure" and "over reactivity"

The path contains the following chain of elementary facts: "decrease in temperature pressure" - "change [increase]" - "mean temperature of heat carrier" - "result from [lead to]" - "formation of excess reactivity" - "includes the entity" - "excess reactivity" - "includes an entity" - "formation of excess reactivity" - "be the result of [lead to]" - "reduction of electric power of Baltic NPP unit".

"Reading" of the graph allows to conclude that an increase in steam pressure in the steam generator will lead to decrease in steam generator temperature pressure, which will lead to an increase in the average temperature of the heat carrier, which will lead to an excess reactivity. The absorption of excess reactivity will lead to a decrease in the electrical capacity of the power unit of the Baltic NPP.

Thus, a connection has been established between the parameters of the vapor pressure in the steam generator and the electric power of the power unit, and the nature of the connection has been established - an increase in pressure will lead to a decrease in power.

Next, it is possible to interactively change the layout of the vertices (see Fig. 8), remove the extra vertices and arcs and save the result, thus forming a completed fact on the problem under consideration. In the future, it could be consulted and used for textual navigation. In particular, the use of a reference to a completed fact in the cognitive rubricator [31] makes it possible to implement the principle of preserving and accumulating knowledge. 


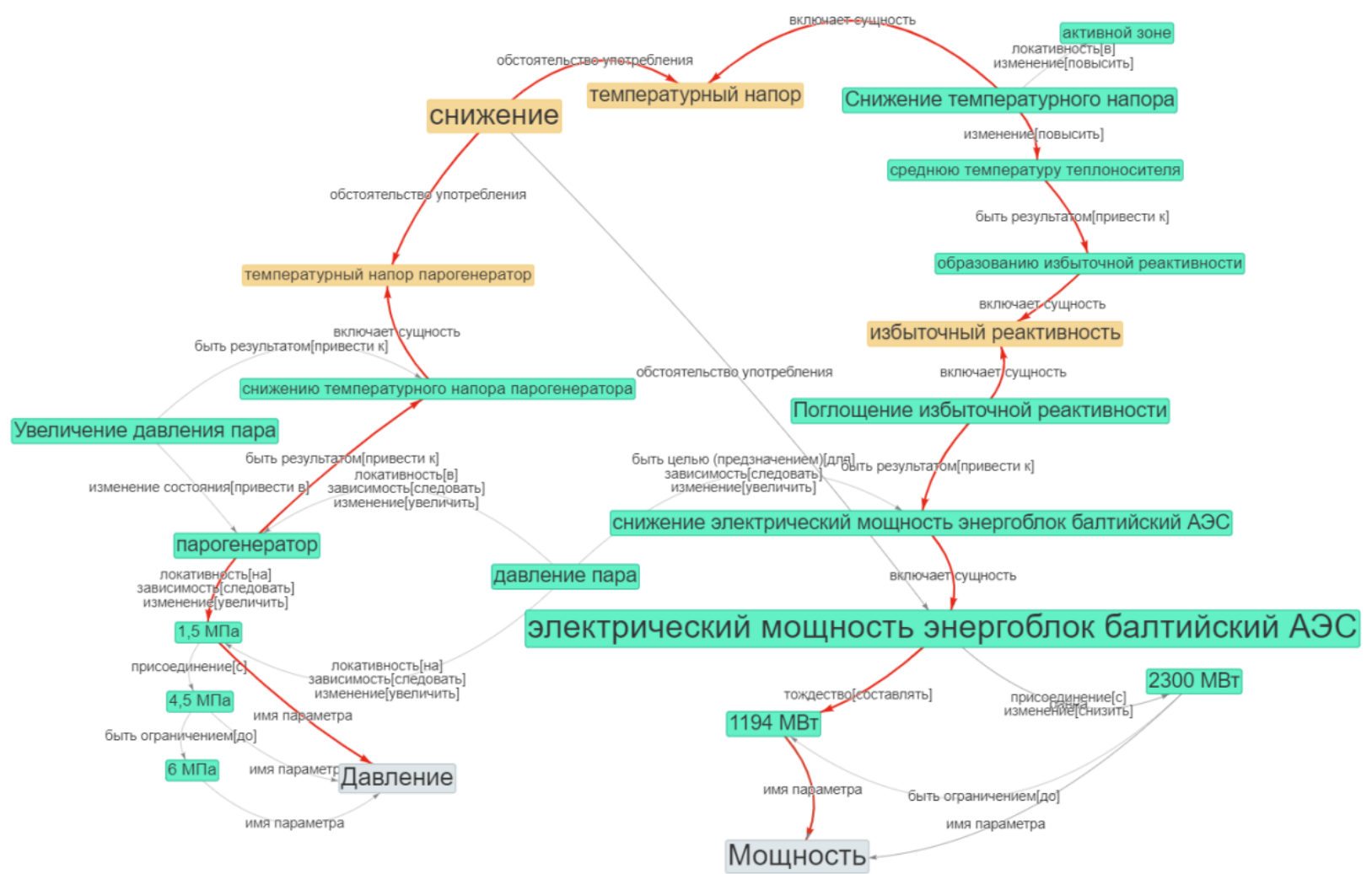

Figure 8 - Fragment of the ontology graph of the text "The problem of incomplete capacity utilization of the Baltic NPP" with interactive vertex layout

\subsection{Neighborhood Analysis}

Let us illustrate the search by the scheme "neighborhood analysis". Let's build a graph based on a text fragment of the design documentation document "Main circulation pumping unit" (MCPU), containing a description of the pump design. Let' s carry out the selection of vertices by the name of the entity "MCPU" and build a neighborhood of the vertex "MCPU" radius 1 . We get the subgraph shown in Fig. 9.

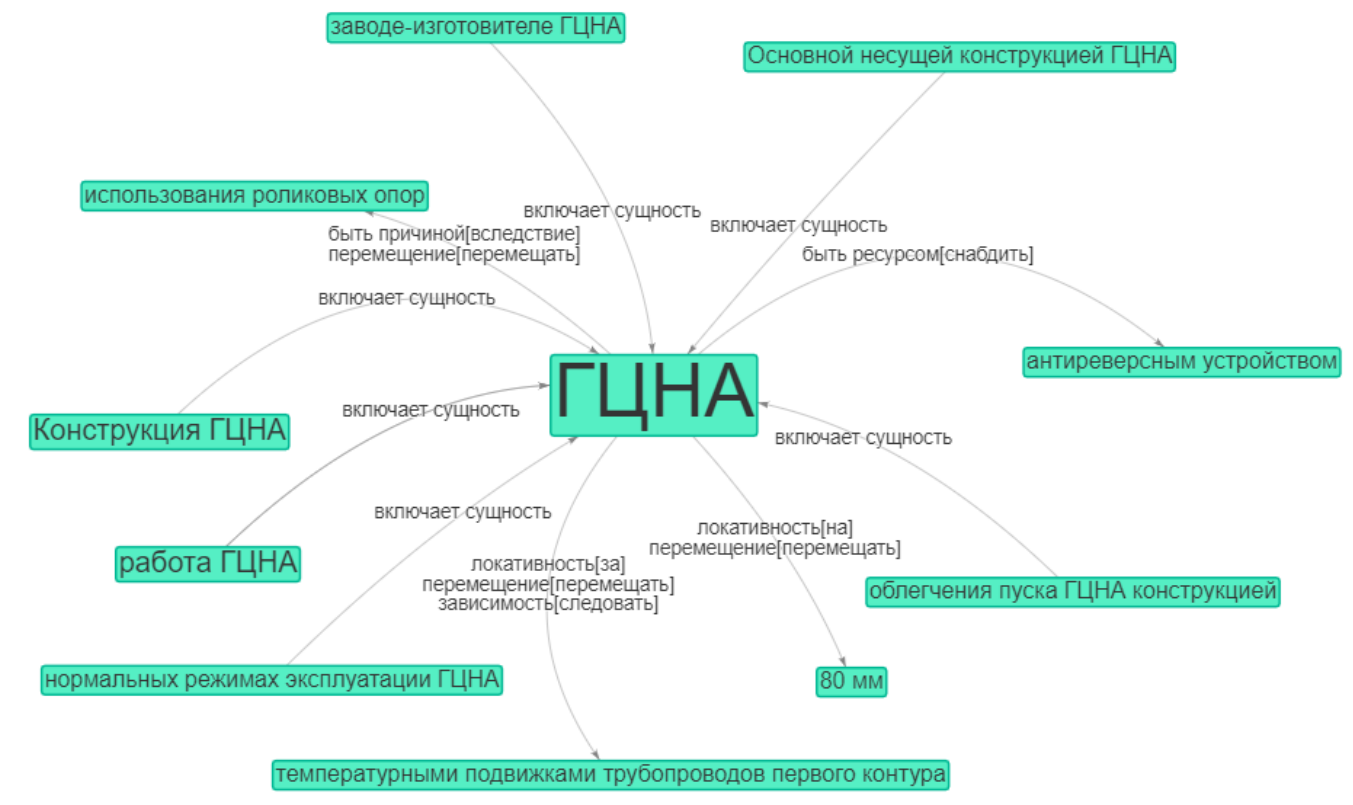

Figure 9 - Neighborhood of the vertex "MCPU" 
All vertices of the formed subgraph (Fig. 9) have the origin of "from the text", as indicated by the vertices' color. The adjacent vertices for the vertex "MCPU" have the same font size (the size depends on the weight), which indicates the equivalence of entity names in the text.

For the elementary fact "anti-reverse device" - "to be a resource [provide]" - "MCPU", build a neighborhood of the vertex "anti-reverse device" (Fig. 10).

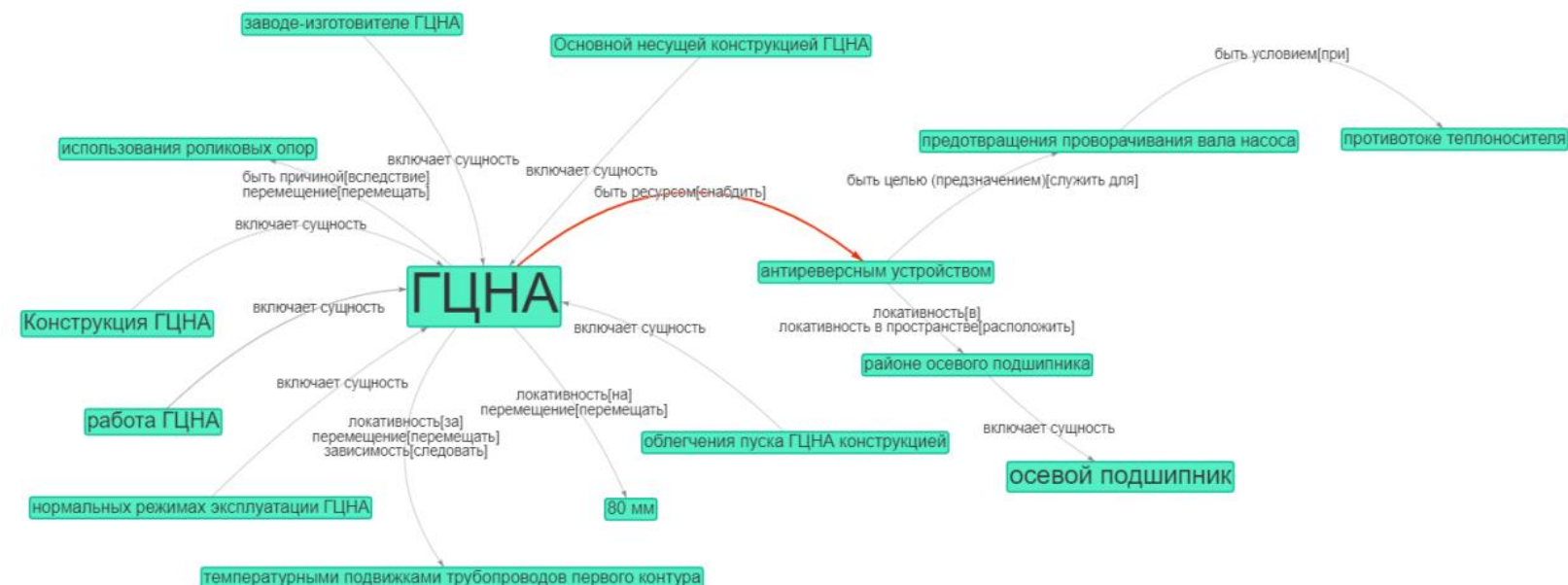

Figure 10 - Expansion of the neighborhood of the term "MCPU" in the direction of "antireverse device"

The built neighborhood includes two chains of elementary facts representing the location and purpose of the anti-reverse device:

— "anti-reverse device" - "to be the goal (purpose) [ serve for]" - "prevent the pump shaft from being rolled up" - "to be a condition [at]" - "counter flow of the heat carrier";

— "anti-reverse device" - "locality [in]" - "area of the axial bearing" - "includes the entity" - "axial bearing".

The vertex of the "the axial bearing area" is linked by the structural-linguistic relationship "includes the entity" with the vertex of the "axial bearing". Moreover, the "axial bearing" vertex is distinguished by its large size in relation to adjacent ones, which signals its greater weight and, accordingly, its significance in the text.

Let us build a situational fact for the elementary fact "area of the axial bearing" "includes an entity" - "axial bearing" by applying the function of constructing a neighborhood to the "axial bearing" vertex. We get the subgraph shown in Fig. 11.

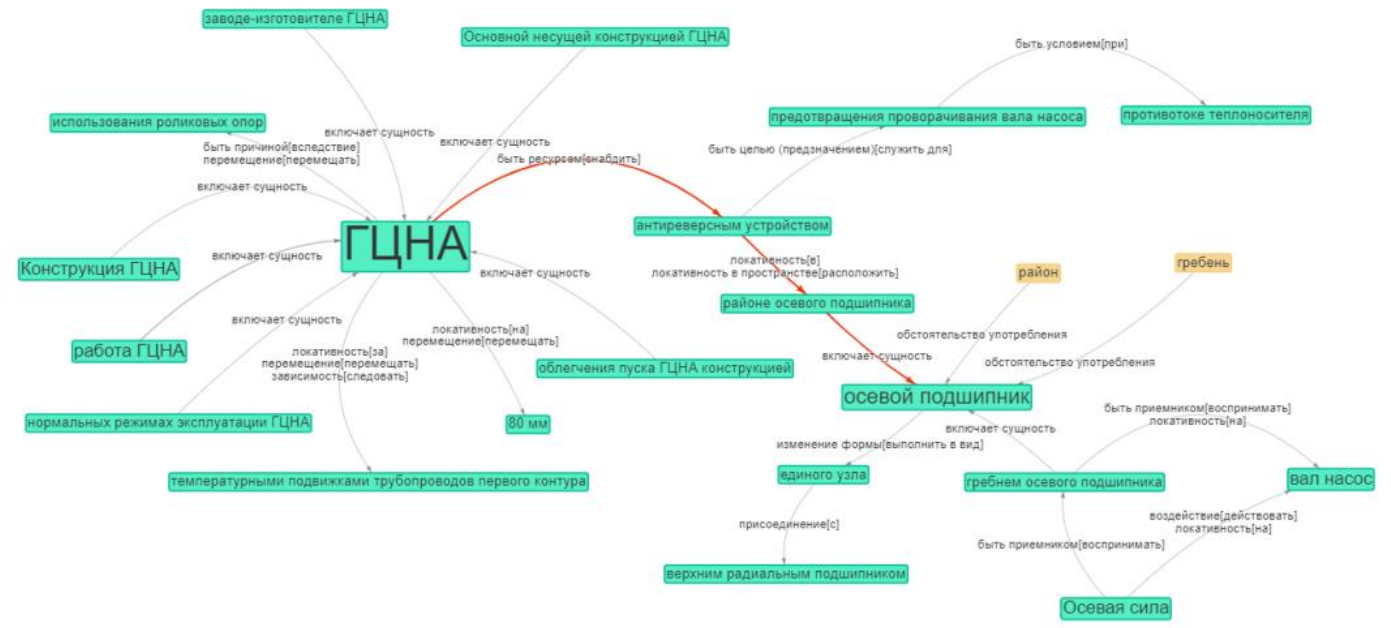

Figure 11 - Example of an expansion of the neighborhood of the term "MCPU" through the neighborhood of the term "anti-reverse device" in the direction of the term "axial bearing" 
The neighborhood of other terms can be studied in a similar way.

Thus, by the constructed graph it is possible to "restore" text: "The MCPU has such a resource as an anti-reverse device, which is designed to prevent the pump shaft from turning under the condition of the carrier counter flow and is located in the area of the axial bearing, which looks like a single unit with an upper radial bearing. In this case, the crest of the axial bearing perceives the axial force which influences on the pump shaft".

Using the function of transition from vertices to the source text, fragments of the text were obtained that were used to build the graph: “... The axial force acting on the pump shaft is perceived by the crest of the axial bearing. The axial bearing is structurally designed in the form of a single unit with an upper radial bearing. ... The MCPU is equipped with an antireverse device, which is used for preventing the pump shaft from turning during counter flow of the heat carrier located in the area of the axial bearing".

A comparison with the original text showed that the meaning was largely not distorted.

\section{Conclusion}

In this article the technology of constructing and visualizing a semantic image of the full text of the document, represented by ontology, is proposed.

The typology of ontology information components by contextual certainty and completeness of the semantic structure ensures the correspondence of the data logical and semantic levels: an elementary fact corresponds to a separate action (event), a situational fact - to a separate statement, and a completed fact - to a decision.

A general scheme for solving information retrieval tasks has been developed, which, in addition to the steps corresponding to the classical information retrieval, involves constructing, visualizing and analyzing the ontology graph of a document. The ontology information retrieval on graphs is reduced to schemes that include finding a chain of facts and searching for the neighborhood of an elementary (situational) fact. The variant graph imaging technology consists of the following steps:

- selection of the elements of a graph in accordance with the user's objective;

- forming a representation according to visualization metaphor;

- forming an image according to the visualization model.

Based on the typology of documentary information retrieval tasks, two visualization metaphors have been defined. The "pathfinding" metaphor corresponds to the construction of a directed chain of facts from the starting points to the target positions. The metaphor "neighborhood analysis" corresponds to the exploration of the environment (context) of the original fact. In general, this makes the perception more effective by means of purposeful and manageable reduction of the dimensions of the operating space and profiling.

The visualization model defines the logic of laying graph elements in the plane. The Barnes-Hut method of laying vertices; placing vertices in the order of use (appearance) of entity names in the text; placing vertices according to the significance of paths (long path or total weight of vertices); laying vertices according to some scheme (for example, the IDEFo functional model) have been implemented.

The developed software allows constructing the ontology graphs on text in natural language, as well as providing opportunities to select, configure and manipulate fragments of ontology according to metaphors of path search and neighborhood analysis. The ontology graph here acts as a technological space of "entry points" into the information array, providing the possibility of a direct transition from the graph vertices to the corresponding fragments of the document text.

The developed interactive tools with ontology graph implement the principle of display dynamism (which provides the possibility of a consistent perception of the object or process), and the tools to reduce the dimensionality of ontology to a level acceptable by human perception - the principle of minimizing the time spent on data analysis. 
At the same time, trial operation and application examples have shown that the quality of visualization largely depends on the quality of constructing a semantic image, in particular on the accuracy of the selection and identification of the entities and relationships in the text.

\section{Acknowledgements}

This work was supported by the Ministry of Science and Higher Education of the Russian Federation (state assignment project No. 0723-2020-0036)

\section{References}

1. Golitsyna, O. L., Maksimov, N. V., Okropishina, O. V., \& Strogonov, V. I. (2012). The ontological approach to the identification of information in tasks of document retrieval. Automatic Documentation and Mathematical Linguistics, 46(3), 125-132.

2. Maksimov, N. V. (2018). The methodological basis of ontological documentary information modeling. Automatic Documentation and Mathematical Linguistics, 52(2), 57-72.

3. Peirce Ch.S., Sowa J. Existential Graphs: MS 514 by Ch. S. Peirce with comment by J.F. Sowa. Retrieved May 20, 2020, from http://www.jfsowa.com/peirce/ms514.htm

4. Mikhailov, A. M., Chernyj, A. I., \& Giljarevskij, R. S. (1968) Fundamentals of Informatics. [in Russian]

5. Zakharova, A., \& Shklyar, A. (2014). Basic principles of data visual models construction, by the example of interactive systems for 3D visualization. Scientific Visualization, 6(2), 62-73. [in Russian]

6. Gordeev, D. S. (2018) A survey of visualization techniques of algorithms on graphs. Scientific Visualization, 10(1), 18-48. [in Russian]

7. Averbukh, V. (2013). Semiotic approach to forming the theory of computer visualization. Scientific Visualization, 5(1), 1-25.

8. Stepin, V. S., Guseynov, A. A., \& Semigin, G. Y. (2010). Novaya filosofskaya entsiklopediya. $\mathrm{V}$ chetyrekh tomakh [Sociology history and modernity]./In-t filosofii RAN. Nauchno-red. M., Mysl, 4, 275-276. [in Russian]

9. Golitsina, O. L., Maksimov, N. V., Okropishina, O. V., \& Strogonov, V. I. (2013). An ontological approach to information identification in tasks of document retrieval: A practical application. Automatic Documentation and Mathematical Linguistics, 47(2), 45-51.

10. Maksimov, N. V., Golitsina, O. L., Monankov, K. V., Lebedev, A. A., Bal, N. A., \& Kyurcheva, S. G. (2019). Semantic Search Tools Based on Ontological Representations of Documentary Information. Automatic Documentation and Mathematical Linguistics, 53(4), 167-178.

11. Skorokhod'ko, E. F. (1974). Linguistic problems of text processing in automated information retrieval systems. Vopr. Inf. Teor. Prakt, (25), 5-120. [in Russian]

12. Belonogov, G. G., Bystrov, I. I., Novoselov, A. P., Kozachuk, M. V., Khoroshilov, A. A., \& Khoroshilov, A. A. (2002). Automatic conceptual text analysis. Automatic Documentation and Mathematical Linguistics, 36(5), 57-65.

13. Maksimov, N. V., Gavrilkina, A. S., Andronova, V. V., \& Tazieva, I. A. (2018). Systematization and identification of semantic relations in ontologies for scientific and technical subject areas. Automatic Documentation and Mathematical Linguistics, 52(6), 306-317.

14. Maksimov, N., Gavrilkina, A., Kuzmina, V., \& Borodina, E. (2020). Ontology of Properties and its Methods of Use: Properties and Unit extraction from texts. Procedia Computer Science, 169, 70-75.

15. Zakharova, A., \& Shklyar, A. (2013). Visualization metaphors. Scientific Visualization, 5(2), 16-24. [in Russian] 
16. Podvesovskii, A. G., \& Isaev, R. A. (2018). Visualization metaphors for fuzzy cognitive maps. Scientific Visualization, 10(4), 13-29.

17. Averbukh, V. L., Bakhterev, M. O., \& Manakov, D. V. (2017). Evaluations of visualization metaphors and views in the context of execution traces and call graphs. Scientific Visualization, 9(5), 1-18.

18. Kasyanov, V., Kasyanova, E. (2014). Information visualization on the base of graph models. Scientific Visualization, 6(1), 31-50. [in Russian]

19. Pupyrev, S. N., \& Tikhonov, A. V. (2010). The analysis of complex networks with dynamic graph visualization. Modelirovanie i Analiz Informatsionnykh Sistem, 17(1), 117-135. [in Russian]

20.Kasyanov, V. N., Zolotukhin, T. A., \& Gordeev, D. S. (2019). Visualization Methods and Algorithms for Graph Representation of Functional Programs. Programming and Computer Software, 45(4), 156-162.

21. Open Source Visualization Library to Display Networks "vis-network.js". Retrieved August 12, 2020, from https://github.com/visjs/vis-network

22. Pilyugin, V. V., \& Milman, I. (2019). Visual analytics and its use in the NRNU MEPhI "Scientific Visualization" laboratory activities. Scientific Visualization, 11(5), 46 - 55.

23. Sugiyama, K. (2002). Graph Drawing and Applications for Software and Knowledge Engineers. Series on Software Engineering and Knowledge Engineering.

24. Apanovich, Z. V. (2012). Modern Force-directed Algorithms for Visualization of Large Volumes of Information. In Problems of Management and Design in Complex Systems (pp. 164-171). [in Russian]

25. Maksimov, N. V., Golitsyna, O. L., Monankov, K. V., \& Gavrilkina, A. S. (2021). A Prototype of a Service for Visual Ontological Analysis of Scientific and Technical Texts. State Registration Certificate, (2021610648). [in Russian]

26. Maksimov, N. V., Golitsyna, O. L., Monankov, K. V., \& Gavrilkina, A. S. (2020). Document Information-Analytical System xIRBIS. State Registration Certificate, (2020661683). [in Russian]

27. Maksimov, N.V., Golitsyna, O. L., Tameev, A.A., Monankov, K. V., Gavrilkina, A. S. et al. (2019). Lexicographic Database for Linguistic Support of Documentary Information Retrieval Tasks. State Registration Certificate, (2019622150). [in Russian]

28.Project AES-2006, JSC "SPbAEP". Retrieved May 20, 2020, from http://atomenergoprom.ru/u/file/npp_2006_rus.pdf [in Russian]

29. Baltic nuclear power plant. Retrieved May 23, 2020, from https://energybase.ru/powerplant/Baltic_NPP [in Russian]

30.Problems of increasing the maneuverability of nuclear power plants. Retrieved May 20, 2020, from https://tesiaes.ru/?p=9250 [in Russian]

31. Maksimov, N. V., Golitsyna, O. L., \& Usenko, A. L. (2014). The structure and components of the operational visual space for scientific interactive information retrieval. Journal on Scietific Visualization, 6(4), 96-106. 